\title{
Validity and Reliability of Hand-Held Dynamometry for Abdominal Flexion Muscular Assessment
}

\author{
Brett D. Tarca, Thomas P. Wycherley, Anthony Meade, Paul Bennett, and Katia E. Ferrar
}

\begin{abstract}
Context: Abdominal musculature underpins core stability, which can allow for optimal performance in many activities of daily living (eg, walking and rising from a chair). Therefore, assessment of the abdominal muscles poses as an important consideration for clinicians in order to identify people at risk of injury or functional decline. Objective: This study aimed to build on the limited amount of knowledge surrounding abdominal muscle strength assessments by investigating the validity and reliability of handheld dynamometry (HHD) for the assessment of isometric abdominal flexion strength. Study Design and Participants: Comparative analysis for validity and test-retest reliability was employed on a cohort of apparently healthy individuals. HHD was compared with the criterion, isokinetic dynamometry, through an isometric contraction of trunk flexion on both instruments. Hand-held dynamometry assessments only were performed on a subsequent day for reliability analysis. The peak values for all assessments were recorded. Results: A total of 35 participants were recruited from the University of South Australia and the general public. Comparative analysis between the HHD and isokinetic dynamometer showed good agreement (intraclass correlation coefficients $=.82$ ), with the Bland-Altman plots confirming no proportional bias. Reliability analysis for the HHD reported good consistency (intraclass correlation coefficients $=.87$ ). Conclusion: HHD together with the participant setup (supine, trunk flexed, and supported at $25^{\circ}$ with the legs horizontal and remaining unfixed) is a valid and reliable tool to assess isometric abdominal flexion strength.
\end{abstract}

Keywords: field test, injury prevention, muscle testing, screening, trunk flexion

Abdominal musculature underpins core stability, which plays a critical role in modulating balance and functional performance. ${ }^{1}$ The abdominal musculature complex also provides protection for vital organs, regulates the internal abdominal pressure, and assists in maintaining posture. ${ }^{2}$

Objective assessment of muscle strength is an important consideration in clinical as well as athletic populations, as it is able to track change in a given muscle or joint movement. ${ }^{3}$ Objective measurement methods can include electromyography, hand-held dynamometry (HHD), and isokinetic dynamometry, with the latter considered the criterion measure for muscle assessment. ${ }^{4,5}$ Isokinetic dynamometers are expensive and of limited use in a field setting since they are not easily portable. ${ }^{4} \mathrm{~A}$ HHD is an easy-to-use, portable, and relatively inexpensive (compared to isokinetic dynamometry) device that is placed between the hand of the assessor and a body segment to determine the isometric muscle force. ${ }^{6}$ A HHD may, therefore, represent a feasible option for coaches and clinicians to assess abdominal flexion muscle strength. Recent evidence suggests that HHD is a valid and reliable method for assessing trunk flexion in a young athletic population that would typically be familiar with undertaking strength assessments as a part of routine training and monitoring, ${ }^{7}$ but these findings cannot be translated to the general population.

The aim of this study was to build on the limited number of studies to date, to investigate the validity and reliability of HHD

\footnotetext{
Tarca, Wycherley, and Ferrar are with the Alliance for Research in Exercise, Nutrition and Activity, University of South Australia, Adelaide, SA, Australia. Tarca is also with the School of Health Sciences, University of South Australia, Adelaide, SA, Australia. Meade and Bennett are with the Central Northern Adelaide Renal Transplantation Service Clinical Research Group, Adelaide, SA, Australia. Tarca (brett.tarca@mymail.unisa.edu.au) is corresponding author.
}

against isokinetic dynamometry to assess isometric abdominal flexion strength in a general population.

\section{Methods}

This study assessed the use of HHD for isometric abdominal flexion strength test-retest reliability and validity (vs isokinetic dynamometry). Ethics approval was provided by the University of South Australia Human Research Ethics Committee (protocol no. 201810), and the study was performed at the University of South Australia from May to July 2019.

\section{Participants}

People aged over 18 years were invited to participate via University of South Australia e-mail distribution lists, flyers, and posters. The exclusion criteria were a current or a history of abdominal-related injury. Written consent was obtained prior to participation.

\section{Study Equipment}

The participants were assessed on 2 separate days within a 7-day period by a single tester, with testing completed at the same time of day. A Lafayette HHD (advanced hand dynamometer; TTM, Inc, Tokyo, Japan) was used. Validity was measured at the first visit using maximum peak torque (in newton meters $[\mathrm{N} \cdot \mathrm{m}]$ ) produced on an isokinetic dynamometer (Cybex NORM ${ }^{\circledR}$; Humac, CA) as the comparator. Reliability was assessed through test-retest using the HHD on the second visit. Height, weight, date of birth, and the moment arm (the vertical distance from the anterior superior iliac spine to the level of the sternal notch) of each participant was recorded prior to testing. 


\section{Participant Setup}

For the purpose of this study, all trunk flexion angles stated are in reference to flexion degrees from horizontal supine.

Figure 1A illustrates the participant setup for the isokinetic dynamometry assessment. Figure 1B illustrates the participant setup for the HHD assessment, which was adapted from previously reported work. ${ }^{7}$

\section{Test Protocol}

Validation. Device assessment order was randomly assigned to the participants. The assessments involved a single warm-up/ familiarization trial, followed by 2 maximal isometric efforts, with the peak torque (isokinetic dynamometry) or kilogram (HHD) value recorded. The participants were asked to, without using their hands to assist, flex their trunk with the objective of trying to curl their body so that their eyes would be aiming toward their navel and continue holding each effort for 3 seconds, exhaling on exertion. A 15-second rest was afforded between efforts, with a 15-minute rest between device changeover.

Reliability. Reliability measures for HHD were completed on the second assessment day.

\section{Data Analysis}

To derive a common metric for analysis, the HHD result in kilograms was transformed to peak torque (in newton meters) by converting to newtons and multiplying by the length (in meters) of the moment arm. Statistical analysis was completed using IMP SPSS statistical software (version 25.0; IBM Corp, Armonk, NY).

\section{Statistical Analyses}

Independent sample $t$ tests were employed post hoc to compare the demographics between the validity cohort and reliability cohort.

Validity. Comparison of the peak torque device values was used to examine agreement between the devices, initially by using a 2way random model with single measure reliability using intraclass correlation coefficients (ICC [2,1]). The ICC reliability rating was poor $<.5$, moderate .5 to .75 , good .75 to .90 , and excellent $>.90 .^{8}$

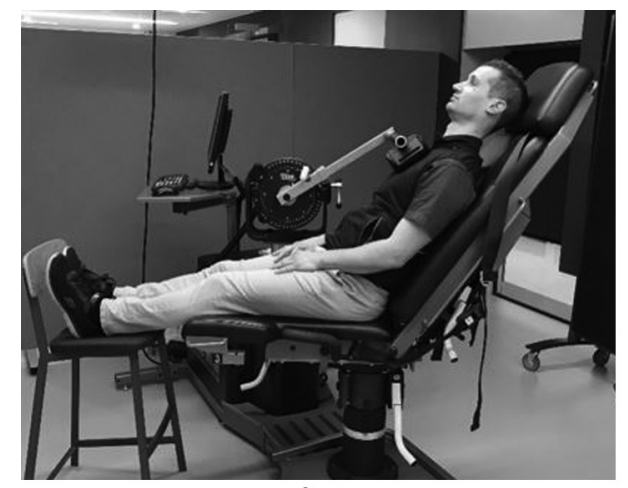

A
Bland-Altman methods were used to confirm validity by analyzing the agreement between measurements, graphically displaying the average difference between measures and the $95 \%$ limits of agreement (mean difference [SD]). ${ }^{9}$ The mean difference and SD were determined through a 1-sample $t$ test. Scatter plots were created with upper and lower limits of agreement calculated. To inform potential proportional bias, linear regression was employed to estimate the association between the average of the 2 measures (independent variable) and the difference between the 2 measures (dependent variable). The $r$ value was interpreted according to Cohen ${ }^{10}$ (effect size: weak $<0.30$, moderate 0.30 0.49 , and strong $>0.5$ ). A $\beta$ coefficient in the regression model as close to 0 as possible with $P>.05$ would confirm no proportional bias.

Reliability. Intratester (tester 1 , between 2 d) reliability of the peak torque values produced on the HHD were assessed using ICC with $95 \%$ confidence intervals using a 2 -way mixed effects model with single measure reliability (ICC $[3,1]$ ). The aforementioned ICC scales were used to classify reliability. ${ }^{8}$ Statistical significance was set at $P<.05$.

\section{Results}

Table 1 describes the participant characteristics. Of the 35 participants that completed validity testing (58\% male), 31 completed reliability testing, with 4 participants unable to complete reliability testing due to reasons unrelated to this study. Mean age and body mass index for this cohort was 33.9 (17.3) years and $24.4(3.6) \mathrm{kg} / \mathrm{m}^{2}$, respectively. Independent sample $t$ tests showed no differences between the validity and reliability cohorts regarding demographics.

\section{Validity}

Agreement between the peak isokinetic dynamometer value and peak HHD value was good ( $\mathrm{ICC}=.82)$, with moderate to excellent rated confidence intervals $(.57-.92){ }^{8}$

A 1-sample $t$ test revealed that the mean difference between instruments differed significantly from zero $(P=.001$; Table 2). The reported mean difference of $8.9(14.6) \mathrm{N} \cdot \mathrm{m}$ (10\% with the

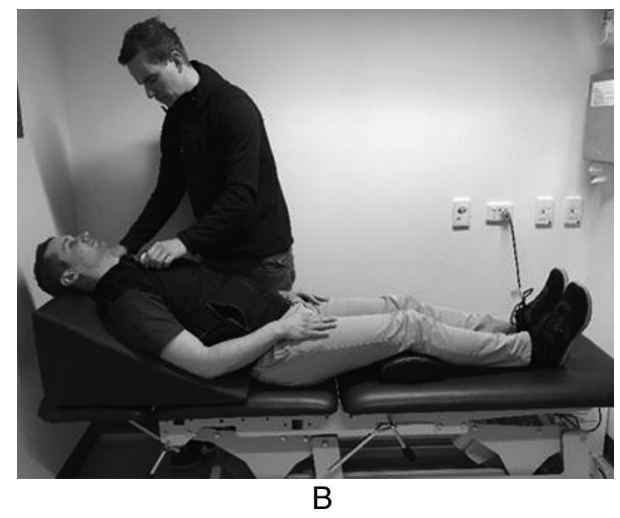

Figure 1 - (A) Isokinetic dynamometry setup with seat adjusted to $55^{\circ}$ trunk flexion and the participant's legs horizontal to the ground, not fixed with feet resting on a stool. The isokinetic dynamometry arm was set centrally over the sternum, with the superior border of the pad set at the height of the sternal notch. Arms were positioned relaxed on the thighs. (B) Hand-held dynamometry setup with a $25^{\circ}$ foam wedge placed under the upper body, beginning at the point of the posterior superior iliac spine. Legs were horizontally on the plinth, not fixed, with a small cushion placed under the knee for support. The hand-held dynamometer was placed centrally over the sternum with the superior border of the hand-held dynamometer at the height of the sternal notch. Arms were positioned relaxed on the thighs. 
isokinetic dynamometer as the reference value) indicates that the isokinetic dynamometer produced higher measurements.

Bland-Altman plots (Figure 2) display the general trend of systematic error found in the 1-sample $t$ test. Further visual inspection and linear regression discovered a weak effect $\left(r=.27\right.$, adjusted $\left.r^{2}=.046\right)$, indicating that the mean values are unrelated and only explain $4.6 \%$ of the variance mean measurement differences. The regression slope did not differ greatly from zero $(\beta=0.15 ; P=.12)$, confirming no proportional bias, with the $95 \%$ limits of agreement ranging from 37.5 to $-19.7 \mathrm{~N} \cdot \mathrm{m}$.

Table 1 Participant Characteristics Mean and SD Separated by Cohorts That Completed Validity and Reliability Testing, Respectively

\begin{tabular}{lccc}
\hline Variable & $\begin{array}{c}\text { Validity } \\
(\mathbf{n = 3 5 )}\end{array}$ & $\begin{array}{c}\text { Reliability } \\
(\mathbf{n = 3 1 )}\end{array}$ & $\boldsymbol{P}$ \\
\hline Male, \% & 57 & 52 & \\
Age, y & $33.9(17.3)$ & $35.2(18.0)$ & .80 \\
Height, $\mathrm{m}$ & $1.75(0.1)$ & $1.74(0.9)$ & \\
Weight, $\mathrm{kg}$ & $75.5(16.7)$ & $73.8(14.4)$ & \\
Body mass index, $\mathrm{kg} / \mathrm{m}^{2}$ & $24.4(3.6)$ & $24.1(3.1)$ & .64 \\
\hline
\end{tabular}

\section{Reliability}

Intrarater reliability was good ( $\mathrm{ICC}=.86)$, with good to excellent confidence intervals (.72-.93). ${ }^{8}$ The standard error of measurement was $4.7 \mathrm{~N} \cdot \mathrm{m}(6.4 \%$ with the HHD day one mean as the reference value).

\section{Discussion}

This study found that, while there was systematic validity error between the HHD and the isokinetic dynamometer measures $(10 \%$ mean difference with isokinetic dynamometry producing higher values), there was good overall agreement between the 2 devices, with no identifiable proportional bias. The HHD showed good to excellent reliability. Based on these results, the HHD represents a valid and reliable tool to assess isometric abdominal flexion strength.

The results from this study are similar in magnitude to the results by De Blaiser et al. ${ }^{7}$ They investigated the validity of HHD for the assessment of trunk flexion in a $30^{\circ}$ supported trunk flexed supine position and reported a mean difference of $8.78(23.85) \mathrm{N} \cdot \mathrm{m}(6 \%$ with the isokinetic dynamometer as the reference value) between instruments, with $95 \%$ limits of agreement at 55.93 and $-37.97 \mathrm{~N} \cdot \mathrm{m}$, which indicates that the protocol used in the present study produced relatively more consistent results. Reliability of the present study protocol was found to be good to excellent, which is consistent with previous work by De Blaiser et $\mathrm{al},{ }^{7}$ and completed in similar body positioning with the same instrument.

Table 2 Descriptive Statistics of Isokinetic Dynamometry and HHD Measurements and Results of a 1-Sample $t$ Test for Comparison of the Mean Difference (Isokinetic Dynamometry - HHD and HHD Day 1 - HHD Day 2)

\begin{tabular}{lcc}
\hline Instrument & Mean (SD) & Mean difference (SD) \\
\hline Isokinetic dynamometer, $\mathrm{N} \cdot \mathrm{m}$ & $86.0(29.3)$ & $8.9(14.6)$ \\
$\mathrm{HHD}, \mathrm{N} \cdot \mathrm{m}$ & $77.1(25.7)$ & $1.0(12.6)$ \\
$\mathrm{HHD}$ day $1, \mathrm{~N} \cdot \mathrm{m}^{\mathrm{a}}$ & $72.9(24.3)$ & .001 \\
HHD day $2, \mathrm{~N} \cdot \mathrm{m}^{\mathrm{a}}$ & $71.9(22.3)$ & \\
\hline
\end{tabular}

${ }^{\mathrm{a}}$ Based on 31 participants who completed reliability testing. $\mathrm{N} \cdot \mathrm{m}=$ newton meters.

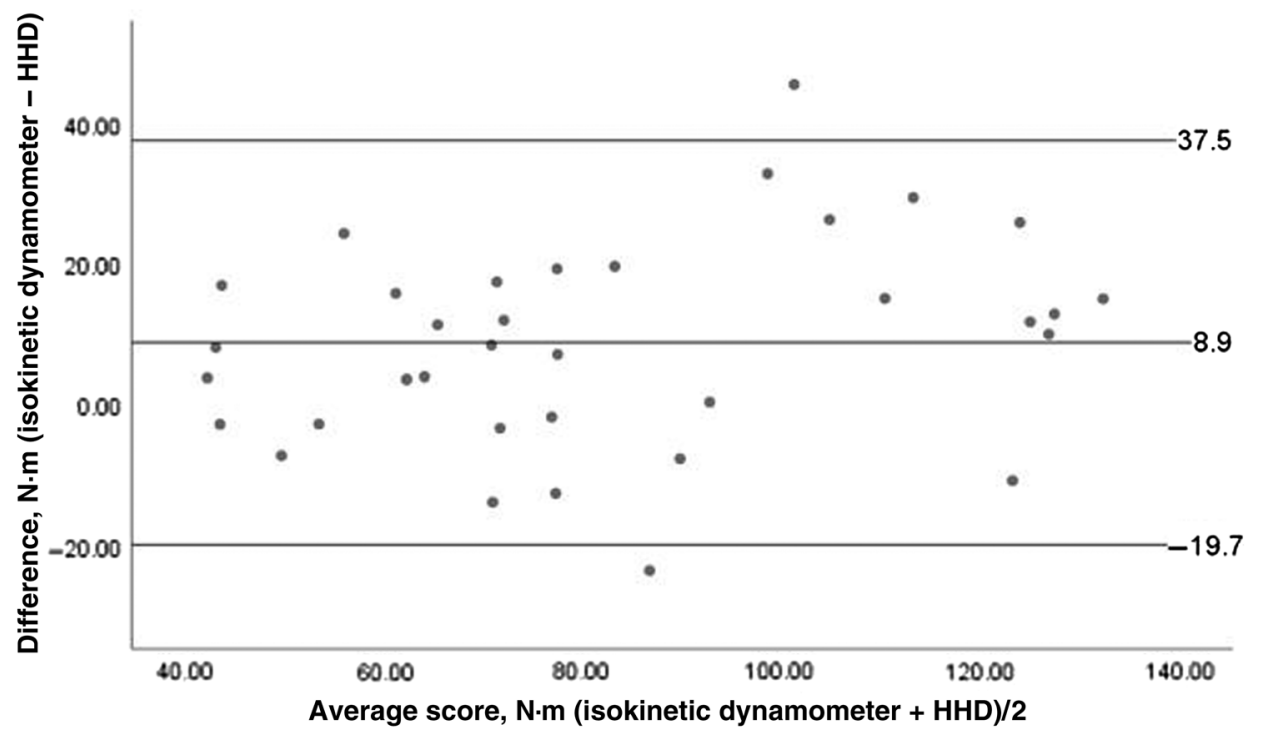

Figure 2 - Bland-Altman plots displaying the average score between the isokinetic dynamometer and the HHD against the difference in scores between instruments. HHD indicates hand-held dynamometer. 
While our findings are consistent with those of De Blaiser et al, ${ }^{7}$ there are key methodological and sample population differences. The current study sampled a population that included university students $(n=15)$, university staff $(n=13)$, and the general population $(n=7)$, whereas the sample of De Blaiser et $\mathrm{al}^{7}$ was limited to a young, sporting, healthy athletic population that is relatively less generalizable. The protocol De Blaiser et $\mathrm{al}^{7}$ employed for the isokinetic dynamometer adopted a standing position with the trunk flexed at $115^{\circ}\left(25^{\circ}\right.$ flexion from vertical) and the lower limbs fixed. This contrasts with the current study, which adopted an upright position with the trunk in a supported $55^{\circ}$ trunk flexion and the legs horizontal in an unfixed position (Figure 1A). This position was employed for 2 reasons; first, it has been shown for limb dynamometry that gravity-assisted measurements can lead to an overestimation of peak torque, ${ }^{11}$ and second, the constraints of the isokinetic dynamometer adjustment options was limited to $55^{\circ}$ flexion and did not allow for a complete replication of the HHD participant setup. Finally, in the current study, the lower limbs were not fixed using a strap; electromyography studies have confirmed that this may decrease the intensity of the rectus abdominis and facilitate activation of hip flexors. ${ }^{12-14}$ An objective of this study was to establish a simple, easy-to-replicate, isometric abdominal flexion strength assessment, with the lower limbs unfixed, a more practical approach in a field test setting, and a more realistic movement type in free living.

It is well established that abdominal musculature underpins core musculature, which is an important facilitator of daily tasks (eg, rising from a chair). ${ }^{1}$ Impaired abdominal strength has been shown as a risk factor for the development of musculoskeletal injuries, which may render individuals unable to perform many activities of daily living. ${ }^{15}$ Therefore, the assessment and continual monitoring of abdominal strength presents an important consideration for clinicians.

The present work provides clinicians with a valid and reliable method; however, its clinical utility is yet to be investigated. Future work should be directed to investigate the predictive validity of this assessment (ie, the ability to predict the onset of unfavorable health outcomes over time and validity in various clinical populations).

An important consideration is the systematic error that was reported in this study (10\% higher values produced on the isokinetic dynamometer). Clinicians requiring absolute strength values may not find this assessment suitable for that purpose. However, for the purpose of monitoring and surveillance to identify changes over time, this assessment is both valid and reliable.

\section{Conclusion}

The results from this study suggest that HHD, with appropriate participant positioning, is a valid and reliable method for assessing and monitoring changes in isometric abdominal flexion strength, which may assist in the earlier detection and/or prevention of functional decline or injury. Future work could be directed at investigating the predictive validity of this test and validity in specific clinical populations.

\section{Acknowledgments}

The authors would like to thank Dr Terry Boyle, a statistician at the University of South Australia who assisted with the statistical analyses and interpretation. No funding sources were involved with this study. The authors declare that they have no conflicts or competing interests.

\section{References}

1. Granacher U, Gollhofer A, Hortobagyi T, Kressig RW, Muehlbauer $\mathrm{T}$. The importance of trunk muscle strength for balance, functional performance, and fall prevention in seniors: a systematic review. Sports Med. 2013;43(7):627-641. PubMed ID: 23568373 doi:10. 1007/s40279-013-0041-1

2. Glenn JM, Galey M, Edwards A, Rickert B, Washington TA. Validity and reliability of the abdominal test and evaluation systems tool (ABTEST) to accurately measure abdominal force. $J$ Sci Med Sport. 2015;18(4):457-462. PubMed ID: 25024133 doi:10.1016/j.jsams. 2014.06.006

3. Carlsson H, Rasmussen-Barr E. Clinical screening tests for assessing movement control in non-specific low-back pain. A systematic review of intra- and inter-observer reliability studies. Man Ther. 2012;18(2):103110. PubMed ID: 23018080 doi:10.1016/j.math.2012.08.004

4. Stark T, Walker B, Phillips JK, Fejer R, Beck R. Hand-held dynamometry correlation with the gold standard isokinetic dynamometry: a systematic review. PM R. 2011;3(5):472-479. PubMed ID: 21570036 doi:10.1016/j.pmrj.2010.10.025

5. Chamorro C, Armijo-Olivo S, De la Fuente C, Fuentes J, Javier Chirosa L. Absolute reliability and concurrent validity of hand held dynamometry and isokinetic dynamometry in the hip, knee and ankle joint: systematic review and meta-analysis. Open Med. 2017;12:359-375. PubMed ID: 29071305 doi:10.1515/med-2017-0052

6. Schrama PP, Stenneberg MS, Lucas C, van Trijffel E. Intraexaminer reliability of hand-held dynamometry in the upper extremity: a systematic review. Arch Phys Med Rehabil. 2014;95(12):24442469. PubMed ID: 24909587 doi:10.1016/j.apmr.2014.05.019

7. De Blaiser C, De Ridder R, Willems T, Danneels L, Roosen P. Reliability and validity of trunk flexor and trunk extensor strength measurements using handheld dynamometry in a healthy athletic population. Phys Ther Sport. 2018;34:180-186. PubMed ID: 30366246 doi:10.1016/j.ptsp.2018.10.005

8. Koo TK, Li MY. A guideline of selecting and reporting intraclass correlation coefficients for reliability research. J Chiropr Med. 2016; 15(2):155-163. PubMed ID: 27330520 doi:10.1016/j.jcm.2016.02.012

9. Bland JM, Altman DG. Measuring agreement in method comparison studies. Stat Methods Med Res. 1999;8(2):135-160. PubMed ID: 10501650 doi:10.1177/096228029900800204

10. Cohen J. Statistical Power Analysis for the Behavioral Sciences. Hillsdale, NJ: L. Erlbaum Associates; 1988.

11. Bygott IL, McMeeken J, Carroll S, Story I. Gravity correction in trunk dynamometry: is it reliable? Isokinet Exerc Sci. 2001;9(1):1-9.

12. Monfort-Panego M, Vera-Garcia FJ, Sanchez-Zuriaga D, Sarti-Martinez MA. Electromyographic studies in abdominal exercises: a literature synthesis. J Manipulative Physiol Ther. 2009;32(3):232244. PubMed ID: 19362234 doi:10.1016/j.jmpt.2009.02.007

13. Lipetz S, Gutin B. An electromyographic study of four abdominal exercises. Med Sci Sports. 1970;2(1):35-38. PubMed ID: 4255344

14. Guimaraes AC, Vaz MA, De Campos MI, Marantes R. The contribution of the rectus abdominis and rectus femoris in twelve selected abdominal exercises. An electromyographic study. J Sports Med Phys Fitness. 1991;31(2):222-230. PubMed ID: 1836517

15. Cowley PM, Swensen TC. Development and reliability of two core stability field tests. J Strength Cond Res. 2008;22(2):619-624. PubMed ID: 18550982 doi:10.1519/JSC.0b013e3181634cb4 